\title{
Correction to: The prevalence and health burden of malnutrition in Belgian older people in the community or residing in nursing homes: results of the NutriAction II study
}

Maurits F. J. Vandewoude ${ }^{1}$. Janneke P. van Wijngaarden ${ }^{2} \cdot$ Lieven De Maesschalck $^{3} \cdot$ Yvette C. Luiking $^{2}$. André Van Gossum ${ }^{4}$

Published online: 12 June 2018

(c) The Author(s) 2018

Correction to: Aging Clinical and Experimental Research https://doi.org/10.1007/s40520-018-0957-2

In the original publication, table row alignment was incorrectly formatted for all the tables. The corrected Tables 1,2, 3 and 4 are given below.

The original article can be found online at https://doi.org/10.1007/ s40520-018-0957-2.

Maurits F. J. Vandewoude

maurits.vandewoude@zna.be

1 Department of Geriatrics (ZNA), University of Antwerp, Antwerp, Belgium

2 Nutricia Research, Nutricia Advanced Medical Nutrition, Utrecht, The Netherlands

3 Mobilab, Thomas More University College, Geel, Belgium

4 Nutrition Support Team, Department of Gastroenterology, Hôpital Erasme, ULB, Brussels, Belgium 
Table 1 Characteristics of the nursing home and community dwelling study populations

\begin{tabular}{|c|c|c|c|}
\hline Parameter & $\begin{array}{l}\text { Nursing home } \\
(n=2480)\end{array}$ & $\begin{array}{l}\text { Community dwelling } \\
(n=819)\end{array}$ & $p$ value \\
\hline Age (years) mean \pm SD & $86.3 \pm 6.2$ & $82.7 \pm 6.1$ & $<0.001$ \\
\hline Sex (\% male/female) & $22 / 78$ & $32 / 68$ & $<0.001$ \\
\hline \multicolumn{4}{|l|}{ Presence of comorbidities } \\
\hline Cancer & $171(7 \%)$ & $87(11 \%)$ & 0.001 \\
\hline Chronic heart failure & $285(12 \%)$ & $113(14 \%)$ & 0.079 \\
\hline COPD & $141(6 \%)$ & $50(6 \%)$ & 0.656 \\
\hline Dementia & $1074(43 \%)$ & $113(14 \%)$ & $<0.001$ \\
\hline Depression & $367(15 \%)$ & $47(6 \%)$ & $<0.001$ \\
\hline Diabetes & $440(18 \%)$ & $163(20 \%)$ & 0.165 \\
\hline Fractures & $313(13 \%)$ & $63(8 \%)$ & $<0.001$ \\
\hline Parkinson & $140(6 \%)$ & $37(5 \%)$ & 0.214 \\
\hline Rheumatoid arthritis & $122(5 \%)$ & $116(14 \%)$ & $<0.001$ \\
\hline Stroke & $284(12 \%)$ & $59(7 \%)$ & 0.001 \\
\hline Number of comorbidities mean \pm SD & $1.5 \pm 1.1$ & $1.1 \pm 0.98$ & $<0.001$ \\
\hline 0 & $407(16 \%)$ & $208(25 \%)$ & \\
\hline 1 & $1035(42 \%)$ & $391(48 \%)$ & \\
\hline 2 & $647(26 \%)$ & $147(18 \%)$ & \\
\hline 3 & $282(11 \%)$ & $55(7 \%)$ & \\
\hline 4 & $85(3 \%)$ & $10(1 \%)$ & \\
\hline 5 & $18(0.7 \%)$ & $7(0.9 \%)$ & \\
\hline 6 & $6(0.2 \%)$ & $1(0.1 \%)$ & \\
\hline $\mathrm{BMI}\left(\mathrm{kg} / \mathrm{m}^{2}\right) \pm \mathrm{SD}$ & $24.3 \pm 5.4$ & $26.3 \pm 5.3$ & $<0.001$ \\
\hline Nutritional status (MNA-SF) & & & $<0.001$ \\
\hline$\%$ malnourished & $336(14 \%)$ & $56(7 \%)$ & \\
\hline$\%$ at risk of malnutrition & $1214(49 \%)$ & $241(29 \%)$ & \\
\hline$\%$ normal & $907(37 \%)$ & $519(63 \%)$ & \\
\hline Weight loss last 3 months & & & $<0.001$ \\
\hline No weight loss & $1541(62 \%)$ & $601(73 \%)$ & \\
\hline $1-3 \mathrm{~kg}$ & $474(19 \%)$ & $88(11 \%)$ & \\
\hline $3-6 \mathrm{~kg}$ & $143(6 \%)$ & $37(5 \%)$ & \\
\hline$>6 \mathrm{~kg}$ & $47(2 \%)$ & $28(3 \%)$ & \\
\hline Unknown & $275(11 \%)$ & $65(8 \%)$ & \\
\hline Use of ONS & $204(8 \%)$ & $14(2 \%)$ & $<0.001$ \\
\hline Being able to climb stairs & $391(16 \%)$ & $268(33 \%)$ & $<0.001$ \\
\hline Being able to walk outside for $5 \mathrm{~min}$ & $853(34 \%)$ & $425(52 \%)$ & $<0.001$ \\
\hline Katz ADL score & & & $<0.001$ \\
\hline Cat $\mathrm{O}$ & $460(19 \%)$ & $228(28 \%)$ & \\
\hline Cat A & $346(14 \%)$ & $214(26 \%)$ & \\
\hline Cat B & $242(10 \%)$ & $188(23 \%)$ & \\
\hline Cat C & $327(13 \%)$ & $129(16 \%)$ & \\
\hline Cat $\mathrm{D}(\mathrm{D}=$ demented $)$ & $242(10 \%)$ & $5(0.6 \%)$ & \\
\hline Cat C_D (fully dependent) & $863(35 \%)$ & $55(7 \%)$ & \\
\hline
\end{tabular}

Data are presented as $n(\%)$, except for age, sex, number of comorbidities, and BMI. Participants had no more than 6 comorbidities. Nutritional status was missing in $n=26$ (CD: $n=3$, NH: $n=23$ )

$A D L$ activities of daily living, $B M I$ body mass index, $C O P D$ chronic obstructive pulmonary disease, $M N A$ $S F$ mini nutritional assessment—short form, $O N S$ oral nutritional supplement 
Table 2 Characteristics of community dwelling study population $(n=819)$, by nutritional status (based on MNA-SF)

\begin{tabular}{|c|c|c|c|c|}
\hline Parameter & $\begin{array}{l}\text { Malnourished }(n=56, \\
7 \%)\end{array}$ & $\begin{array}{l}\text { At risk of malnutrition } \\
(n=241,29 \%)\end{array}$ & $\begin{array}{l}\text { Normal nutritional status } \\
(n=519,63 \%)\end{array}$ & $p$ value \\
\hline Age (years) mean \pm SD & $83.0 \pm 6.0$ & $83.4 \pm 6.0$ & $82.3 \pm 6.1$ & 0.063 \\
\hline Sex (\% male/female) & $32 / 68$ & $29 / 71$ & $34 / 66$ & 0.376 \\
\hline Number of comorbidities mean \pm SD & $1.4 \pm 0.9$ & $1.4 \pm 1.0$ & $1.0 \pm 0.9$ & $<0.001$ \\
\hline $\mathrm{BMI}\left(\mathrm{kg} / \mathrm{m}^{2}\right)$ mean $\pm \mathrm{SD}$ & $20.2 \pm 3.5$ & $24.4 \pm 5.1$ & $27.9 \pm 4.8$ & $<0.001$ \\
\hline Weight loss last 3 months & & & & $<0.001$ \\
\hline No weight loss & $3(5 \%)$ & $119(49 \%)$ & $476(92 \%)$ & \\
\hline $1-3 \mathrm{~kg}$ & $9(16 \%)$ & $47(20 \%)$ & $32(6 \%)$ & \\
\hline $3-6 \mathrm{~kg}$ & $18(32 \%)$ & $19(8 \%)$ & 0 & \\
\hline$>6 \mathrm{~kg}$ & $15(27 \%)$ & $13(5 \%)$ & 0 & \\
\hline Unknown & $11(20 \%)$ & $43(18 \%)$ & $11(2 \%)$ & \\
\hline Use of ONS & $4(7 \%)$ & $8(3 \%)$ & $2(0.4 \%)$ & $<0.001$ \\
\hline Being able to climb stairs & $10(18 \%)$ & $49(20 \%)$ & $208(40 \%)$ & $<0.001$ \\
\hline Being able to walk outside for 5 min & $19(34 \%)$ & $97(40 \%)$ & $306(59 \%)$ & $<0.001$ \\
\hline Katz ADL score & & & & $<0.001$ \\
\hline Cat $\mathrm{O}$ & $6(11 \%)$ & $36(15 \%)$ & $186(36 \%)$ & \\
\hline Cat A & $11(20 \%)$ & $50(21 \%)$ & $153(30 \%)$ & \\
\hline Cat B & $14(25 \%)$ & $71(30 \%)$ & $101(20 \%)$ & \\
\hline Cat C & $13(23 \%)$ & $53(22 \%)$ & $63(12 \%)$ & \\
\hline Cat $\mathrm{D}(\mathrm{D}=$ demented $)$ & 0 & $1(0.4 \%)$ & $4(1 \%)$ & \\
\hline Cat C_D (fully dependent) & $12(21 \%)$ & $30(12 \%)$ & $12(2 \%)$ & \\
\hline
\end{tabular}

Data are presented as $n(\%)$, except for age, sex, number of comorbidities, and BMI. Nutritional status was missing in $n=3$; results may therefore not add up to $100 \%$

$A D L$ activities of daily living, $B M I$ body mass index, $M N A-S F$ mini nutritional assessment—short form, $O N S$ oral nutritional supplement

Table 3 Characteristics of the nursing home study population $(n=2480)$, by nutritional status (based on MNA-SF)

\begin{tabular}{|c|c|c|c|c|}
\hline Parameter & $\begin{array}{l}\text { Malnourished }(n=336, \\
14 \%)\end{array}$ & $\begin{array}{l}\text { At risk of malnutrition } \\
(n=1214,49 \%)\end{array}$ & $\begin{array}{l}\text { Normal nutritional status } \\
(n=907,37 \%)\end{array}$ & $p$ value \\
\hline Age (years) mean $\pm \mathrm{SD}$ & $86.7 \pm 6.4$ & $86.7 \pm 6.2$ & $85.6 \pm 6.2$ & $<0.001$ \\
\hline Sex (\% male/female) & $22 / 78$ & $20 / 80$ & $23 / 77$ & 0.258 \\
\hline Number of comorbidities mean \pm SD & $1.9 \pm 1.2$ & $1.6 \pm 1.1$ & $1.1 \pm 1.0$ & $<0.001$ \\
\hline $\mathrm{BMI}\left(\mathrm{kg} / \mathrm{m}^{2}\right) \pm \mathrm{SD}$ & $19.7(3.9)$ & $23.2(4.8)$ & $27.4(4.7)$ & $<0.001$ \\
\hline Weight loss last 3 months & & & & $<0.001$ \\
\hline No weight loss & $34(10 \%)$ & $679(56 \%)$ & $817(90 \%)$ & \\
\hline $1-3 \mathrm{~kg}$ & $85(25 \%)$ & $307(25 \%)$ & $77(9 \%)$ & \\
\hline $3-6 \mathrm{~kg}$ & $101(30 \%)$ & $39(3 \%)$ & 0 & \\
\hline$>6 \mathrm{~kg}$ & $39(12 \%)$ & $8(1 \%)$ & 0 & \\
\hline Unknown & $77(23 \%)$ & $181(15 \%)$ & $13(1 \%)$ & \\
\hline Use of ONS & $73(22 \%)$ & $111(9 \%)$ & $19(2 \%)$ & $<0.001$ \\
\hline Being able to climb stairs & $10(3 \%)$ & $130(11 \%)$ & $246(27 \%)$ & $<0.001$ \\
\hline Being able to walk outside for $5 \mathrm{~min}$ & $36(11 \%)$ & $310(26 \%)$ & $496(55 \%)$ & $<0.001$ \\
\hline Katz ADL score & & & & $<0.001$ \\
\hline Cat $\mathrm{O}$ & $13(4 \%)$ & $133(11 \%)$ & $312(34 \%)$ & \\
\hline Cat A & $13(4 \%)$ & $113(9 \%)$ & $219(24 \%)$ & \\
\hline Cat B & $31(9 \%)$ & $126(10 \%)$ & $85(9 \%)$ & \\
\hline Cat $\mathrm{C}$ & $58(17 \%)$ & $175(14 \%)$ & $92(10 \%)$ & \\
\hline Cat $\mathrm{D}(\mathrm{D}=$ demented $)$ & $15(5 \%)$ & $112(9 \%)$ & $109(12 \%)$ & \\
\hline Cat C_D (fully dependent) & $206(61 \%)$ & $555(46 \%)$ & $90(10 \%)$ & \\
\hline
\end{tabular}

Data are presented as $n(\%)$, except for age, sex, number of comorbidities, and BMI. Nutritional status is missing in $n=23$; results may therefore not add up to $100 \%$

$A D L$ activities of daily living, $B M I$ body mass index, $M N A-S F$ mini nutritional assessment—short form, $O N S$ oral nutritional supplement 
Table 4 Nutritional status determined with MNA-SF of community dwelling older adults and nursing home residents, by their comorbidities

\begin{tabular}{|c|c|c|c|}
\hline & Malnourished & At risk of malnutrition & Normal nutritional status \\
\hline Community dwelling $(n=816)$ & $n=56(7 \%)$ & $n=241(29 \%)$ & $n=519(63 \%)$ \\
\hline \multicolumn{4}{|l|}{ Presence of comorbidities } \\
\hline Cancer $(n=87)$ & $8(9 \%)$ & $33(38 \%)$ & $46(53 \%)$ \\
\hline Chronic heart failure $(n=113)$ & $6(5 \%)$ & $41(36 \%)$ & $66(58 \%)$ \\
\hline $\operatorname{COPD}(n=50)$ & $8(16 \%)$ & $16(32 \%)$ & $26(52 \%)$ \\
\hline Dementia $(n=110)$ & $16(15 \%)$ & $59(54 \%)$ & $35(32 \%)$ \\
\hline Depression $(n=44)$ & $8(18 \%)$ & $22(50 \%)$ & $14(32 \%)$ \\
\hline Diabetes $(n=162)$ & $5(3 \%)$ & $44(27 \%)$ & $113(70 \%)$ \\
\hline Fractures $(n=62)$ & $4(7 \%)$ & $24(39 \%)$ & $34(55 \%)$ \\
\hline Parkinson $(n=37)$ & $3(8 \%)$ & $18(49 \%)$ & $16(43 \%)$ \\
\hline Rheumatoid arthritis $(n=116)$ & $5(4 \%)$ & $40(35 \%)$ & $71(61 \%)$ \\
\hline Stroke $(n=59)$ & $4(7 \%)$ & $18(31 \%)$ & $37(63 \%)$ \\
\hline Nursing home $(n=2457)$ & $n=336(14 \%)$ & $n=1214(49 \%)$ & $n=907(37 \%)$ \\
\hline \multicolumn{4}{|l|}{ Presence of comorbidities } \\
\hline Cancer $(n=169)$ & $28(17 \%)$ & $74(44 \%)$ & $67(40 \%)$ \\
\hline Chronic heart failure $(n=282)$ & $32(11 \%)$ & $150(53 \%)$ & $100(36 \%)$ \\
\hline $\operatorname{COPD}(n=141)$ & $25(18 \%)$ & $67(48 \%)$ & $49(35 \%)$ \\
\hline Dementia $(n=1051)$ & $212(20 \%)$ & $649(62 \%)$ & $190(18 \%)$ \\
\hline Depression $(n=344)$ & $81(24 \%)$ & $192(56 \%)$ & $71(21 \%)$ \\
\hline Diabetes $(n=437)$ & $49(11 \%)$ & $208(47 \%)$ & $180(41 \%)$ \\
\hline Fractures $(n=310)$ & $58(19 \%)$ & $146(47 \%)$ & $106(34 \%)$ \\
\hline Parkinson $(n=139)$ & $29(21 \%)$ & $71(51 \%)$ & $39(28 \%)$ \\
\hline Rheumatoid arthritis $(n=122)$ & $20(16 \%)$ & $59(48 \%)$ & $43(35 \%)$ \\
\hline Stroke $(n=284)$ & $38(13 \%)$ & $147(52 \%)$ & $99(35 \%)$ \\
\hline
\end{tabular}

Data are presented as $n(\%)$. Data represent nutritional status categorized per comorbidity (row percentage). The list of scored comorbidities was longer, reported comorbidities represent comorbidities with prevalence $>5 \%$. Numbers do not add up because comorbidities overlap (column totals)

$C O P D$ chronic obstructive pulmonary disease

Open Access This article is distributed under the terms of the Creative Commons Attribution 4.0 International License (http://creativeco mmons.org/licenses/by/4.0/), which permits unrestricted use, distribution, and reproduction in any medium, provided you give appropriate credit to the original author(s) and the source, provide a link to the Creative Commons license, and indicate if changes were made. 